\title{
Behavioral Interventions and Pharmacotherapies for Cannabis Use Disorder
}

Aaron J. Bobb, $M D^{1,2, *}$
Kevin P. Hill, MD, $M H S^{1,2}$

\author{
Address \\ ${ }^{*}$ Division of Alcohol and Drug Abuse, McLean Hospital, 115 Mill Street, Belmont, \\ MA 02478, USA \\ abobb@partners.org \\ ${ }^{2}$ Department of Psychiatry, Harvard Medical School, Boston, MA, USA
}

Published online: 12 March 2014

(C) Springer International Publishing AG 2014

Keywords Marijuana - Cannabis use disorder - Substance use disorders - Treatment - Pharmacotherapy • Behavioral interventions - Motivational interviewing · Motivational enhancement therapy · Cognitive behavioral therapy · Contingency management · Multidimensional family therapy · Emerging treatments

\section{Opinion statement}

Millions of Americans develop cannabis use disorder, which requires treatment to prevent numerous associated adverse outcomes. There are several evidence-based behavioral interventions, including motivational interviewing, motivational enhancement therapy, cognitive behavioral therapy, contingency management, and multidimensional family therapy, all with moderate effectiveness. None of the behavioral interventions are clearly superior to any of the others, whether in combination or alone. Thus, individual patient and therapist factors, such as patient preference and therapist expertise, should determine which is used. Current$l y$, there are no FDA-approved medications for treatment of cannabis use disorder. Some pharmacologic agents have shown promise in early studies, most notably n-acetylcysteine and gabapentin, but none have been replicated. Given that gabapentin and buspirone have been widely used in psychiatric populations and have relatively few side effects, either could be considered as an augmenting agent to behavioral interventions, but neither should be continued for more than 12 weeks unless there is clear evidence of reduction in use. Evidence suggests venlafaxine and naltrexone are not effective in reducing use.

\section{Introduction}

Cannabis is the most commonly used illicit drug in the world [1] and in the United States [2]. It was used at least once by $42 \%$ of all Americans and $45 \%$ of 12 th graders $[3,4 \bullet \bullet]$. Potency of the active ingredient in marijuana, $\delta$-9-tetrahydrocannabinol (THC), has increased by a factor of six to seven from 1970 to 2010 [5]. Increased potency raises the risk of transitioning from use to addiction: $8-9 \%$ of adults and $14-16 \%$ of adolescents who use will become dependent [6]. 
As the decriminalization, legalization, and medical marijuana movements have progressed in recent years, perceived risk has decreased and use has increased. Among 12th graders, the proportion endorsing the perception of great risk in smoking marijuana occasionally decreased from $26 \%$ to $20.6 \%$ from 2008 to 2012; while the proportion using marijuana in the past year increased from $32.4 \%$ to $36.4 \%$ [4••].

Cannabis use disorder (see Table 1 for DSM-5 criteria) is associated with numerous adverse outcomes, such as impaired cognition, poor educational attainment, unemployment, risky sexual behaviors, early pregnancy, crime, progression to further drug use, development of psychotic disorders, and withdrawal syndrome $[8,9 \bullet]$. Eighteen percent of all substance use admissions are for primary cannabis dependence, an increase from $15 \%$ in 2001 [10].

Systematic research on treating marijuana abuse and dependence began with psychosocial interventions in the 1980s and pharmacologic interventions in the 2000s [11], including motivational interviewing, motivational enhancement therapy, cognitive behavioral therapy, contingency management, multidimensional family therapy, and medications such as n-acetyl cysteine, gabapentin, buspirone, dronabinol, and others. However, guidelines for treating cannabis use disorder are sparse. Below is a summary of available treatment options with the evidence supporting their use.

\section{Treatment}

\section{Pharmacologic agents}

- There are no FDA-approved medications for the treatment of cannabis dependence. Below are medications which have been studied in trials with at least 20 participants. Thus, not included below are studies of baclofen $(n=10)$ [12], bupropion sustained release (SR) $(n=10)$ [13], divalproex $(n=7)$ [14], mirtazapine $(n=11)$ [12], and nefazodone $(n=7)$ [15]. See Table 2 for a summary of medications listed below.

\section{Agents with evidence suggesting reduced marijuana use in patients with cannabis use disorder}

\section{$\mathrm{N}$-acetylcysteine}

- N-acetylcysteine is an n-acetyl prodrug of the amino acid cysteine, and is thought to be involved in glutamate neurotransmission [16].

- Gray and colleagues [17] studied n-acetylcysteine (1200 mg divided into two oral doses daily) in an 8-week, randomized, double-blind, placebo-controlled trial, with 116 older adolescents with cannabis dependence. They found a statistically higher likelihood of a negative urine toxicology test among those taking n-acetylcysteine versus those taking placebo at the end of the study ( $40.9 \%$ vs $27.2 \%$, $p=$ $0.03)$ and a non-significant trend at 1-month follow up (19.0\% vs $10.3 \%, p=0.13)$. There were no serious adverse events associated with n-acetylcysteine use.

Standard dosage given the experimental nature of the use of this agent for this disorder, there is no known standard dosage. 


\section{Table 1. DSM-5 criteria for cannabis use disorder}

A problematic pattern of cannabis use, with two or more of the following.

- Cannabis is taken in larger amounts, or over a longer period, than was intended

- Persistent desire, or unsuccessful efforts, to cut down or control use

- A great deal of time is spent in activities necessary to obtain, use, or recover from cannabis

- Craving, or a strong desire to use

- Recurrent use resulting in a failure to fulfill major role obligations at work, school, or home

- Continued use despite persistent or recurrent social or interpersonal problems caused, or exacerbated, by the effects of cannabis

- Tolerance: either a need for markedly increased amounts to achieve intoxication or desired effect, or a markedly diminished effect with continued use of the same amount

- Withdrawal: either the characteristic cannabis withdrawal syndrome, or cannabis is used to relieve or avoid withdrawal symptoms

The symptoms occur within a 12-month period and cause clinically significant impairment or distress.

Classify as mild (two or three symptoms), moderate (four or five symptoms), or severe (six or more symptoms).

DSM-5 criteria for cannabis use disorder [7].

Contraindications relatively few. Some serious anaphylactoid reactions have been reported with both intravenous and oral administration [18].

Main drug interactions no known interactions.

Main side effects hypotension, tachycardia, angioedema, pruritis, urticaria, nausea, vomiting, and bronchospasm $[18,19]$.

Cost inexpensive.

Gabapentin

- Gabapentin is an alkylated analog of gamma-amino-butyric acid (GABA), FDA approved for the treatment of seizures and post-herpetic neuralgia. It is believed to modulate GABAergic mechanisms by blocking the $\alpha-2 \mathrm{~d}$ subunit of the voltage-gated calcium channel [20].

- Mason and colleagues [21] studied gabapentin (1200 mg divided into three oral doses daily) in a 12-week, randomized, double-blind, placebo-controlled, phase IIa trial, in 50 adults with cannabis dependence. They found statistically significant reductions in grams used and days of use in participants receiving gabapentin, along with reductions in cannabis withdrawal symptoms, marijuana cravings, sleep problems, and depression scores, with improvements in mea-

\section{Table 2. Medications for cannabis use disorder}

\section{Evidence}

Evidence suggests that the medication leads to reduced marijuana use

Evidence does not suggest that the medication leads to reduced marijuana use

\section{Medication}

$\mathrm{N}$-acetylcysteine, gabapentin, buspirone, dronabinol

Nefazodone, bupropion SR, divalproex, venlafaxine, naltrexone 


\section{Buspirone}

Standard dosage

Contraindications

Main drug interactions

Main side effects

Cost sures of executive functioning. There were no serious adverse events associated with gabapentin use. However, both those receiving gabapentin and those receiving placebo had dramatic reductions in use (less than 0.5 grams of marijuana use per week, and less than 1 day of use per week, at the end of the study).

given the experimental nature of the use of this agent for this disorder, there is no known standard dosage.

no absolute contraindications.

may enhance the effect of other CNS depressants.

dizziness, drowsiness, ataxia, and fatigue, among others.

expensive.

- Busprione is a non-benzodiazepine anxiolytic, FDA approved for the treatment of generalized anxiety disorder.

- McRae-Clark and colleagues [22] studied buspirone (60 mg daily, orally) in a 12-week, randomized, double-blind, placebo-controlled trial, in 50 adults with cannabis dependence. They found a trend showing a higher percentage of negative drug screens among those receiving buspirone than among those receiving placebo $(29 \%$ vs $11 \%, p=0.07)$; the trend was significant when only examining the 24 participants who completed the trial $(p=0.01)$. There were no group differences in withdrawal symptoms.

Standard dosage given the experimental nature of the use of this agent for this disorder, there is no known standard dosage.

Contraindications no absolute contraindications.

Main drug interactions may enhance the effect of other CNS depressants. Monitor for serotonin syndrome if given with other serotonergic medications.

Main side effects dizziness, drowsiness, and headache.

Cost expensive.

- Dronabinol is an orally bioavailable synthetic form of THC, which is the main psychoactive component of marijuana acting at the cannabinoid 1 receptor [23]. It is approved for AIDS-related anorexia and chemotherapy-associated nausea and vomiting refractory to other antiemetics.

- Levin and others [23] studied dronabinol (40 mg divided into two oral doses daily) in a 12-week, randomized, double-blind, placebocontrolled trial, in 156 adults with cannabis dependence. They found no significant difference in abstinence at weeks 7 and $8(17.7 \%$ and $15.6 \%$ for dronabinol and placebo, respectively), although they did find higher treatment retention (77\% vs $61 \%$, respectively) and lower withdrawal symptoms in the dronabinol group. There were four serious adverse events, but none was deemed to be study related. 
Standard dosage given the experimental nature of the use of this agent for this disorder, there is no known standard dosage.

Contraindications hypersensitivity to marijuana, cannabinoids, or sesame seed oil.

Main drug interactions may enhance the effect of other CNS depressants.

Main side effects euphoria, abnormal thinking, dizziness, paranoia, somnolence, nausea, vomiting, among others.

Cost expensive.

\title{
Agents without evidence suggesting reduced marijuana use in patients with cannabis use disorder
}

\author{
Nefazodone and bupropion SR
}

- Nefazodone is a serotonin and norepinephrine reuptake inhibitor and a serotonin 2a receptor antagonist, FDA approved for the treatment of depression. Bupropion is a norepinephrine and dopamine reuptake inhibitor, FDA approved for the treatment of depression and for smoking cessation.

- Carpenter and colleagues [24] studied nefazodone (600 mg divided into two oral doses daily) and bupropion SR (300 mg divided into two oral doses daily) in a 13-week, randomized, double-blind, placebo-controlled trial, in 116 adults with cannabis dependence. They found no difference in abstinence or withdrawal symptoms among those receiving nefazodone, bupropion SR, or placebo.

- Divalproex is an anticonvulsant with effects upon GABA transmission, approved for the treatment of seizures and bipolar disorder, as well as for migraine prophylaxis.

- Levin and colleagues [25] studied divalproex (up to $2000 \mathrm{mg}$ daily, orally, with target plasma concentrations of $50-120 \mathrm{ng} / \mathrm{mL}$ ) in a 6week, randomized, placebo-controlled trial, in 25 adults with cannabis dependence. They found no difference in use rates among those receiving divalproex or placebo.

- Venlafaxine is a serotonin and norepinephrine reuptake inhibitor, FDA approved for the treatment of major depressive disorder, generalized anxiety disorder, social anxiety disorder, and panic disorder.

- Levin and colleagues [26] studied venlafaxine extended release (XR) (up to $375 \mathrm{mg} /$ day, orally) in a 12-week, randomized, double-blind, placebo-controlled trial, with 103 adults with comorbid cannabis dependence and major depressive disorder or dysthymia. They found less abstinence in those receiving venlafaxine versus placebo $(11.8 \%$ and $36.5 \%$, respectively, $p<0.01$ ). 
- Naltrexone is an opioid $\mu$ antagonist, FDA approved for treatment of alcohol and opioid dependence.

- Cooper and Haney [27] studied the interaction of naltrexone $(0,12$, 25,50 , and $100 \mathrm{mg}$, orally) with smoked marijuana (0 or $3.27 \%$ THC) in a within-subject, randomized, double-blind trial, with 29 adults who used marijuana daily. They found that naltrexone alone (with $0 \%$ THC) reduced ratings of 'liking', 'take again', and 'stimulated;' but, when used with $3.27 \%$ THC, naltrexone increased ratings of 'liking', 'take again', 'stimulated', 'high', 'good', and 'strength.' They concluded that naltrexone may increase the abuse liability of cannabinoids.

- Haney and colleagues [28] studied the interaction of naltrexone (0 or $50 \mathrm{mg}$, orally) with oral THC in a within-subject, randomized, double-blind trial, in 23 adults who smoked marijuana heavily. They found that naltrexone increased the positive subjective effects and reinforcing effects of THC without altering THC plasma levels.

\section{Behavioral interventions}

- Motivational interviewing (MI), motivational enhancement therapy (MET), cognitive behavioral therapy (CBT), contingency management (CM), multidimensional family therapy (MDFT), and combinations thereof are all effective in the treatment of cannabis use disorder. Most comparison studies do not find significant differences between treatments. In studies of treatment-seeking patients, all interventions lead to reductions in use of between 20-60\% after shortterm therapy, with less effect at long-term follow up (see below). In a meta-analysis, Dutra and colleagues [29] found an effect size of 0.81 [95\% CI 0.25-1.36] for psychosocial interventions of marijuana dependence, which compared favorably with studies of all substance use disorders (effect size 0.45 [0.27-0.63]). The mean dropout rate in studies of psychosocial treatment for cannabis dependence was $28 \%$ and the mean abstinence rate was $26 \%$.

\section{Brief motivational interviewing (MI) and motivational enhancement therapy (MET)}

- $\mathrm{MI}$ is a conversational, person-centered, collaborative, goal-oriented style of communication, with special emphasis on the language of change. It is designed to address ambivalence about change and increase motivation for change. The four processes of MI are engaging, 
focusing, evoking, and planning; the core skills include open-ended questions, affirming, reflecting listening, summarizing, and informing and advising; and the core values of MI are partnership, acceptance, compassion, and evocation [30]. Motivational enhancement therapy (MET) is an approach to therapy based on the principles of MI, typically involving one to four 45- or 90-minute sessions [11].

- Several studies have shown reduction in use after only two sessions of MI or MET

- Walker and colleagues [31] compared two 1-hour sessions of MET with educational feedback in 310 non-treatment-seeking adolescents. They found more reduction in use in the MET group than in the educational feedback group at 3 months ( $21 \%$ vs $8 \%$ ) but not at 12 months (16\% vs $9 \%$ ).

- Stein and colleagues [32] compared two sessions of MI with an assessment-only group in 332 non-treatment-seeking young women who regularly used marijuana. They found less use in the MI group than in the assessment-only group at 3 months (OR $0.53, p=0.01$ ) but not at 1 or 6 months. Among participants expressing some desire to quit, those in the MI group were less likely to use at all three time points (OR 0.42, OR 0.31 , and OR 0.35 for 1,3 , and 6 months, respectively; $p<0.05$ ).

- Stephens and colleagues [33] compared two sessions of MI with educational feedback in 188 adult marijuana users who were ambivalent about change. They found more reduction in use in the MI group than in the educational feedback group at 7 weeks (18\% vs $6 \%$ ) and 1 year (19\% vs $4 \%$ ).

- Stephens and colleagues [34] compared two sessions of MI with 14 sessions of CBT in 291 adult marijuana users. They found significant reductions in use in both groups at 4 months (67\% and $74 \%)$ and 16 months ( $46 \%$ and $52 \%$ ), without significant differences between groups. Both were superior to the waitlist group (which had a $31 \%$ reduction in use at 4 months).

- CBT links thoughts, emotions, and behaviors in an interactional model. As applied to substance use disorders, CBT aims to develop and enhance coping strategies, including exploring the consequences of use, self-monitoring, recognizing and coping with triggers and cravings, and identifying situations that could lead to use [35]. Typical courses of CBT for substance abuse consist of 6-14 hour-long sessions [11].

- Most studies of CBT for cannabis dependence include CBT as part of a larger intervention (e.g., CBT plus contingency management), though some studies investigate CBT alone.

- Hendriks and others [36] compared CBT with MDFT in 109 adolescents with cannabis dependence. They found no difference in reduction in use between groups at 6 months (31\% and $36 \%$ ) or 
12 months ( $24 \%$ and $32 \%$ ). In post-hoc analyses, they found than adolescents aged 17 and 18 years and those without coexisting psychiatric problems benefited more from CBT; while younger adolescents and those with conduct disorder, oppositional defiant disorder, or internalizing problems benefited more from MDFT.

- Liddle and colleagues [37] compared CBT with MDFT in 224 adolescents with cannabis dependence or abuse. They found no difference in reduction in use at 3 months $(17 \%$ and $51 \%)$ or 12 months ( $46 \%$ and $59 \%$ ). However, MDFT was found to be superior in decreasing drug abuse problem severity.

\section{Multidimensional family therapy (MDFT)}

- MDFT includes sessions with adolescents and their parents individually and together, addressing personal and interpersonal issues, family functioning, parenting skills, family monitoring, and limit setting, as well as specific strategies for finding alternatives to drug use [38].

- See the section on CBT for evidence showing MDFT is generally equivalent to CBT for treating cannabis dependence.

- $\mathrm{CM}$ is a behavioral approach based on operant conditioning in which patients are given rewards (typically, vouchers or cash) contingent upon targeted outcomes (e.g., providing negative urine samples, attending therapy sessions, or doing CBT homework). Typically, the value of the reward increases for each consecutive desired behavior and reverts back to the original, lower value if the patient engages in the undesired behavior.

- Litt and colleagues [39] compared CM for being abstinent with CM for completing homework in 215 adults with marijuana dependence, all of whom received MET plus CBT. Also included was a case management control condition. All groups improved, including the case management control condition, with an increase in abstinent days from about $10 \%$ to $50 \%$, with no differences between groups in use, abstinence, or marijuana-related problems.

- Stanger and colleagues [40] compared abstinence-based CM with attendance-based CM in 69 adolescents with problematic marijuana use who all received combination MET plus CBT. Participants in both groups had reductions in use from $45-55 \%$ of days using marijuana (at baseline), to 9-12 \% (at 14 weeks), to 15-30\% (at 9 months), with less use at all time points for those receiving abstinence-based CM. Those receiving abstinence-based CM were also more likely to have 8 or more weeks of abstinence ( $53 \%$ vs $30 \%$, $p=$ 0.06 ) and 10 or more weeks of abstinence ( $50 \%$ vs $18 \%, p=0.01$ ).

- Carroll and colleagues [41] compared MET plus CBT with or without $\mathrm{CM}$ with drug counseling with or without CM in 136 young adults 
with cannabis dependence. Adding CM to either MET plus CBT or drug counseling resulted in significantly longer durations of continuous abstinence and significantly more negative urine samples. They also found that those in the MET plus CBT group reduced their use over time as compared with those in the drug counseling group.

\section{Combinations of MI, MET, CBT, CM, and MDFT}

- Given that MI, MET, CM, CBT, and MDFT have all been found to be effective, many recent studies have investigated whether combining two or more modalities is more effective than using any one particular modality. No clear consensus emerges: some studies find no differences, while other studies offer conflicting evidence as to which combination or single modality treatment is best.

- Budney and colleagues [42] compared CBT alone, CBT plus CM, and $\mathrm{CM}$ alone, in 90 adults with cannabis dependence. They found reductions in use in all groups at 14 weeks $(67 \%, 61 \%$, and $56 \%$, respectively) and 1 year (30\%, $50 \%, 30 \%$, respectively), with evidence for more continuous abstinence with $\mathrm{CM}$ alone compared with CBT alone at 14 weeks, no difference between CM alone and CM plus CBT at 14 weeks, and better effects for CBT plus CM than either treatment alone at 1 year.

- Carroll and colleagues [43] compared CBT alone, CBT plus CM (for abstinence, attendance, and homework completion), and CM alone, in 127 adults with cannabis dependence. At 12 weeks, they found a reduction in use in all groups, ranging from $29 \%$ to $63 \%$, highest for those getting CBT alone. The reductions tended to last: at 1-year follow up, use was down by between 35 and $50 \%$ from baseline values in all groups. Surprisingly, they found worse outcomes among those getting CBT plus CM for negative drug screens than among those getting $\mathrm{CM}$ alone.

- Kadden and colleagues [44] compared CM, MET plus CBT, MET plus CBT plus CM, and case management control in 240 adults with cannabis dependence. All groups had reduced use, from $85-95 \%$ of days used at baseline to 35-55\% (at 2 months) to 40-55\% (at 1 year). The CM-only group had the highest abstinence rates at 2 months; the MET plus CBT plus CM group had the highest abstinence rates at follow up.

- The Marijuana Treatment Project Research Group [45] compared two sessions of MET with nine sessions of a combination of MET plus CBT with a waitlist condition, in 450 adults with cannabis dependence. The nine-session group reduced use by $59 \%$ and $50 \%$ (at 4 and 9 months, respectively), which was greater than the two-session group (35\% and $31 \%$, respectively), and the waitlist group (16\% at 4 months).

- Dennis and colleagues [46] compared (i) five sessions of MET plus CBT, (ii) 12 sessions of MET plus CBT, (iii) family education and therapy, (iv) adolescent community reinforcement approach, and (v) MDFT in 600 adolescents with cannabis dependence or abuse. They 


\section{Conclusion}

found similar reductions in use in all groups, with an average of $24 \%$ increase in days abstinent.

- Hoch and colleagues [47] compared a combination of CBT plus MET plus problem-solving training with a waitlist group in 279 adults with cannabis dependence. Those receiving active treatment had significantly improved rates of abstinence ( $46 \%, 32 \%$, and $36 \%$ at 10,22 , and 36 weeks, respectively) compared with those in the waitlist group ( $18 \%$ at 10 weeks).

- Gates and colleagues [48] compared four 1-hour telephone sessions of MI plus CBT with a waitlist group in 110 adults with cannabis dependence. Those receiving the intervention reduced their use more than those in the waitlist group at both 4 weeks (62\% vs $38 \%$ ) and 12 weeks (68\% vs $44 \%$ ).

Clinicians working with patients with cannabis use disorder should first assess their patient's motivation to reduce or abstain from using. If a patient is not interested in treatment or is ambivalent, evidence suggests that as little as two sessions of MI or MET can still lead to reductions in use [31-33]. If a patient is seeking treatment, any of the behavioral interventions described above, including MI, MET, CBT, CM, and MDFT, alone or in combination, can lead to moderate reductions in use. Clinicians should consider adding medication, such as n-acetylcysteine, gabapentin, buspirone, or dronabinol, to augment behavioral interventions, while being aware that none are FDA approved and all require replication of evidence showing reduction in use.

\section{Compliance with Ethics Guidelines}

\section{Conflict of Interest}

This work has been supported by NIDA grant K99RO0DA029115 granted to Kevin Hill.

Aaron J. Bobb declares that he has no conflict of interest.

Human and Animal Rights and Informed Consent

This article does not contain any studies with human or animal subjects performed by any of the authors.

\section{References and Recommended Reading}

Papers of particular interest, published recently, have been highlighted as:

- Of importance

- Of major importance

1. UN Office on Drugs \& Crime. World Drug Report 2011. United Nations Publications. Sales No.

2. Substance Abuse and Mental Health Services AdE.11.XI.10, Vienna, Austria; 2011. ministration. Results from the 2008 National Survey on Drug Use and Health: National Findings. Office 
of Applied Studies, NSDUH Series H-36, HHS Publication No. SMA 09-4434, Rockville, MD; 2009.

3. Substance Abuse and Mental Health Services Administration. Results from the 2010 National Survey on Drug Use and Health: Mental Health Findings. NSDUH Series H-42, HHS Publication No. (SMA) 11-4667, Rockville, MD; 2012.

4.• Johnston LD, O'Malley PM, Bachman JG, Schulenberg JE. Monitoring the future national results on adolescent drug use: overview of key findings, 2011. Ann Arbor: Institute for Social Research, The University of Michigan; 2012. Results of annual survey that document increased use of marijuana among young people along with declining perception of risk.

5. Sevigny EL. Is today's marijuana more potent simply because it's fresher? Drug Test Anal. 2013;5(1):62-7.

6. Anthony JC. The epidemiology of cannabis dependence. In: Roffman RA, Stephens RS, editors. Cannabis dependence: its nature, consequences and treatment. International Research Monographs in the Addictions. Cambridgeshire: The University Press; 2006. p. 58-105.

7. American Psychiatric Association. Diagnostic and statistical manual of mental disorders. 5th ed. Arlington, VA: American Psychiatric Publishing; 2013.

8. Nordstrom BR, Levin FR. Treatment of cannabis use disorders: a review of the literature. Am J Addict. 2007;16(5):331-42.

9. $\quad$ Danovitch I, Gorelick DA. State of the art treatments for cannabis dependence. Psychiatr Clin N Am. 2012;35(2):309-26. Overview of cannabis use, the intoxication and withdrawal syndromes, and emerging pharmacological targets for treating cannabis dependence.

Overview of cannabis use, the intoxication and withdrawal syndromes, and emerging pharmacological targets for treating cannabis dependence

10. Substance Abuse and Mental Health Services Administration, Center for Behavioral Health Statistics and Quality. Treatment Episode Data Set (TEDS): 2001-2011. National admissions to substance abuse treatment services. BHSIS Series S-65, HHS Publication No. (SMA) 13-4722, Rockville, MD; 2013.

11. Budney AJ, Roffman R, Stephens RS, Walker D. Marijuana dependence and its treatment. Addict Sci Clin Pract. 2007;4(1):4-16.

12. Haney M, Hart CL, Vosburg SK, Comer SD, Reed SC, Cooper ZD, et al. Effects of baclofen and mirtazapine on a laboratory model of marijuana withdrawal and relapse. Psychopharmacology (Berlin). 2010;211(2):233-44.

13. Haney M, Ward AS, Comer SD, Hart CL, Foltin RW, Fischman MW. Bupropion SR worsens mood during marijuana withdrawal in humans. Psychopharmacology (Berlin). 2001;155(2):171-9.

14. Haney M, Hart CL, Vosburg SK, Nasser J, Bennett A, Zubaran C, et al. Marijuana withdrawal in humans: effects of oral THC or divalproex. Neuropsychopharmacology. 2004;29(1):158-70.

15. Haney M, Hart CL, Ward AS, Foltin RW. Nefazodone decreases anxiety during marijuana withdrawal in humans. Psychopharmacology (Berl). 2003;165(2):157-65.

16. Kalivas PW, Lalumiere RT, Knackstedt L, Shen H. Glutamate transmission in addiction. Neuropharmacology. 2009;56 Suppl 1:169-73.

17. Gray KM, Carpenter MJ, Baker NL, DeSantis SM, Kryway E, Hartwell KJ, et al. A double-blind randomized controlled trial of N-acetylcysteine in cannabis-dependent adolescents. Am J Psychiatry. 2012;169(8):805-12.

18. Mroz LS, Benitez JG, Krenzelok EP. Angioedema with oral $\mathrm{N}$-acetylcysteine. Ann Emerg Med. 1997;30(2):240-1.

19. Bebarta VS, Kao L, Froberg B, Clark RF, Lavonas E, Qi $M$, et al. A multicenter comparison of the safety of oral versus intravenous acetylcysteine for treatment of acetaminophen overdose. Clin Toxicol (Phila). 2010;48(5):424-30.

20. Sills GJ. The mechanisms of action of gabapentin and pregabalin. Curr Opin Pharmacol. 2006;6(1):10813.

21. Mason BJ, Crean R, Goodell V, Light JM, Quello S, Shadan F, et al. A proof-of-concept randomized controlled study of gabapentin: effects on cannabis use, withdrawal and executive function deficits in cannabis-dependent adults.

Neuropsychopharmacology. 2012;37(7):1689-98.

22. McRae-Clark AL, Carter RE, Killeen TK, Carpenter MJ, Wahlquist AE, Simpson SA, et al. A placebo-controlled trial of buspirone for the treatment of marijuana dependence. Drug Alcohol Depend. 2009;105(1-2):132-8.

23. Levin FR, Mariani JJ, Brooks DJ, Pavlicova M, Cheng W, Nunes EV. Dronabinol for the treatment of cannabis dependence: a randomized, double-blind, placebo-controlled trial. Drug Alcohol Depend. 2011;116(1-3):142-50.

24. Carpenter KM, McDowell D, Brooks DJ, Cheng WY, Levin FR. A preliminary trial: double-blind comparison of nefazodone, bupropion-SR, and placebo in the treatment of cannabis dependence. Am J Addict. 2009;18(1):53-64.

25. Levin FR, McDowell D, Evans SM, Nunes E, Akerele E, Donovan S, et al. Pharmacotherapy for marijuana dependence: a double-blind, placebo-controlled pilot study of divalproex sodium. Am J Addict. 2004;13(1):21-32. 
26. Levin FR, Mariani J, Brooks DJ, Pavlicova M, Nunes $\mathrm{EV}$, Agosti V, et al. A randomized double-blind, placebo-controlled trial of venlafaxine-extended release for co-occurring cannabis dependence and depressive disorders. Addiction. 2013;108(6):1084-94.

27. Cooper ZD, Haney M. Opioid antagonism enhances marijuana's effects in heavy marijuana smokers. Psychopharmacology (Berlin). 2010;211(2):141-8.

28. Haney $M$, Bisaga $A$, Foltin RW. Interaction between naltrexone and oral THC in heavy marijuana smokers. Psychopharmacology (Berlin). 2003;166(1):77-85.

29. Dutra L, Stathopoulou G, Basden SL, Leyro TM, Powers MB, Otto MW. A meta-analytic review of psychosocial interventions for substance use disorders. Am J Psychiatry. 2008;165(2):179-87.

30. Miller WR, Rollnick S. Motivational interviewing: helping people change. 3rd ed. New York, NY: The Guilford Press; 2013.

31. Walker DD, Stephens R, Roffman R, Demarce J, Lozano B, Towe S, et al. Randomized controlled trial of motivational enhancement therapy with nontreatment-seeking adolescent cannabis users: a further test of the teen marijuana check-up. Psychol Addict Behav. 2011;25(3):474-84.

32. Stein MD, Hagerty CE, Herman DS, Phipps MG, Anderson BJ. A brief marijuana intervention for nontreatment-seeking young adult women. J Subst Abus Treat. 2011;40(2):189-98.

33. Stephens RS, Roffman RA, Fearer SA, Williams C, Burke RS. The marijuana check-up: promoting change in ambivalent marijuana users. Addiction. 2007;102(6):947-57.

34. Stephens RS, Roffman RA, Curtin L. Comparison of extended versus brief treatments for marijuana use. J Consult Clin Psychol. 2000;68(5):898-908.

35. National Institute on Drug Abuse. Principles of drug addiction treatment: a research-based guide. 2012, NIH Publication No. 12-4180.

36. Hendriks V, van der Schee E, Blanken P. Treatment of adolescents with a cannabis use disorder: main findings of a randomized controlled trial comparing multidimensional family therapy and cognitive behavioral therapy in The Netherlands. Drug Alcohol Depend. 2011;119(1-2):64-71.

37. Liddle HA, Dakof GA, Turner RM, Henderson CE, Greenbaum PE. Treating adolescent drug abuse: a randomized trial comparing multidimensional family therapy and cognitive behavior therapy. Addiction. 2008;103(10):1660-70.
38. Sherman C. NIDA notes: multidimensional family therapy for adolescent drug abuse offers broad, lasting benefits. www.drugabuse.gov/news-events/nidanotes (2010).

39. Litt MD, Kadden RM, Petry NM. Behavioral treatment for marijuana dependence: randomized trial of contingency management and self-efficacy enhancement. Addict Behav. 2013;38(3):1764-75.

40. Stanger C, Budney AJ, Kamon JL, Thostensen J. A randomized trial of contingency management for adolescent marijuana abuse and dependence. Drug Alcohol Depend. 2009;105(3):240-7.

41. Carroll KM, Easton CJ, Nich C, Hunkele KA, Neavins TM, Sinha R, et al. The use of contingency management and motivational/skills-building therapy to treat young adults with marijuana dependence. J Consult Clin Psychol. 2006;74(5):955-66.

42. Budney AJ, Moore BA, Rocha HL, Higgins ST. Clinical trial of abstinence-based vouchers and cognitive-behavioral therapy for cannabis dependence. J Consult Clin Psychol. 2006;74(2):307-16.

43. Carroll KM, Nich C, Lapaglia DM, Peters EN, Easton CJ, Petry NM. Combining cognitive behavioral therapy and contingency management to enhance their effects in treating cannabis dependence: less can be more, more or less. Addiction. 2012;107(9):1650-9.

44. Kadden RM, Litt MD, Kabela-Cormier E, Petry NM. Abstinence rates following behavioral treatments for marijuana dependence. Addict Behav. 2007;32(6):1220-36.

45. Marijuana Treatment Project Research Group. Brief treatments for cannabis dependence: findings from a randomized multisite trial. J Consult Clin Psychol. 2004;72(3):455-66.

46. Dennis M, Godley SH, Diamond G, Tims FM, Babor T, Donaldson J, et al. The cannabis youth treatment (CYT) study: main findings from two randomized trials. J Subst Abus Treat. 2004;27(3):197-213.

47. Hoch E, Buhringer G, Pixa A, Dittmer K, Henker J, Seifert A, et al. CANDIS treatment program for cannabis use disorders: findings from a randomized multi-site translational trial. Drug Alcohol Depend. 2014;134:185-93.

48. Gates PJ, Norberg MM, Copeland J, Digiusto E. Randomized controlled trial of novel cannabis use intervention delivered by telephone. Addiction. 2012;107(12):2149-58. 